\title{
Using Personas Effectively
}

\author{
Peter Bagnall \\ SurfaceEffect \\ 18a Castle Park \\ Lancaster, UK \\ +44 (0)1524 39145 \\ pete@surfaceeffect.com \\ What is a persona? \\ How to create personas \\ User research \\ Persona generation
}

\begin{abstract}
Personas are a powerful design and communication tool to help all those involved in the creation of interactive systems to better focus their efforts on their users. A persona is a fictional character made to represent an archetypal user, and is best derived from field research. They help direct design, and communicate both to marketing and engineering teams.
\end{abstract}

\section{Keywords}

Personas, Empathy, Design Methodology

\section{TUTORIAL OVERVIEW}

Personas have become an increasingly common tool in the interaction designer's armoury over the last five years. But while many are using personas, few are getting the full benefit of the technique. While using personas may appear straightforward, there are a number of subtleties, which can substantially increase their effectiveness. There are also a number of non-obvious applications, which will help delegates get more from the method.

For any design project, understanding your users is clearly of vital importance. Personas are a highly effective, easy to use, method for ensuring that understanding is infused into the design throughout the development lifecycle.

Peter has substantial experience using personas. He worked for two years at Cooper, Alan Cooper's firm in Silicon Valley, which is credited with inventing the persona methodology. His research also includes persona methodology, specifically examining where the technique breaks down. He has taught the technique on Lancaster Universities MRes course for the last two years.

\section{KEY LEARNING OUTCOMES}

- How to collect relevant data for persona creation

- How to create personas, including from user interview data

- How to use personas to generate and guide an evolving design

- How to use personas to manage client expectations and requirements

- Why personas work, and the limits of their utility

\section{TUTORIAL SCHEDULE}

\subsection{Segment 1 (90 minutes)}

Lecture: Creating Personas

(C) Peter Bagnall, 2007.

Published by the British Computer Society

Volume 2 Proceedings of the 21st BCS HCI Group Conference

HCI 2007, Lancaster University, 3-7 September 2007

Devina Ramduny-Ellis \& Dorothy Rachovides (Editors)
Lecture: Using personas

How are they used (in design, evaluation and communications) Primary, secondary and negative personas, and walk-on roles

Avoiding common design pitfalls

elastic users

self referential

Client Management through Personas

\subsection{Segment 2 (90 minutes)}

Practical exercise: User Research

After a demonstration, delegates will interview each other as a way of collecting a body of user research aimed at designing a Cinema guide system. This interview information will then be collated amongst the group, and between 2 and 4 personas generated from the interview data as group exercise directed by the tutor. A primary persona will then be selected for the exercise in segment 3 .

\subsection{Segment 3 (90 minutes)}

\section{Practical exercise: Persona Guided Design}

In this exercise the design for the Cinema guide system will be derived from the personas. Delegates will be guided through a group exercise (3-4 delegates per group) to create scenarios that aim to address the primary persona's goals. These scenarios will then be used to form a conceptual design of the system, and set of functions the system must support. The emerging design will be tested against the persona to ensure it is appropriate.

\subsection{Segment 4 (90 minutes)}

Lecture: Persona Theory

Why do Personas work?

Persona Overload

The purpose of the Primary Persona

Empathic Distance

Solutions to Persona Overload

Solutions to reduce Empathic Distance

Persona Reuse - Risks

Discussion \& Questions

The last 30 minutes of the day will be reserved for discussions arising out of the exercises, and delegates specific questions about how the technique might be applied in their organisations. 